\title{
Leptospirosis in Sri Lanka, 2003-2012
}

\author{
Suneth B Agampodi ${ }^{1}$ \\ Journal of the Ceylon College of Physicians, 2014, 44, 7-13
}

\section{Introduction}

Leptospirosis is considered as the most widespread zoonotic disease ${ }^{1}$. Increasing number of leptospirosis outbreaks have been reported recently from several countries ${ }^{2}$. One major concern of the disease burden estimates of leptospirosis was the geographical bias of published data that might cause regional gaps. Some of the high endemic countries such as Sri Lanka were not included in the Leptospirosis Epidemiology Reference Group systematic review due to lack of scientifically sound data ${ }^{3}$.

Rajasuriya et al. in first reported confirmed cases of Leptospirosis in Sri Lanka in 19594. In 60's and 70's leptospirosis was shown to be a wide spread disease in Sri Lanka where outbreaks were reported from Kegalle ${ }^{5}$, Ratnapura ${ }^{6,7}$, Ragama $^{8}$ and Colombo ${ }^{9}$. Since 1976 for a period of 30 years scientific publications on leptospirosis was lacking. However, routinely reported data suggests that Sri Lanka has experienced a large outbreak of leptospirosis in 2003 with 2,234 reported cases ${ }^{11}$. In 2008, Sri Lanka experienced the worst ever reported outbreak of leptospirosis ${ }^{12}$ with 7,406 suspected cases and 204 deaths ${ }^{13}$. Sri lanka was having one of the highest incidence worldwide during 2008-12 period with a mean annual incidence of 35.7 per 100,000 population ${ }^{14}$.

The series of work presented here on leptospirosis has been initiated in 2007 with the observation of leptospirosis as the third leading communicable disease in the Medical Officer of Health area Beruwala $^{15}$. An internet based literature search based on this observation showed that there were no published work on leptospirosis in Sri Lanka since 1976 to 2007 except a case report on neuroleptospirosis ${ }^{16}$. By the time Sri Lanka was experiencing the largest reported outbreak of leptospirosis in recent past, the control programme of leptospirosis was hampered by the lack of local data. Even the control strategies used in the programme was shown to be not evidence informed ${ }^{17}$. The primary purpose of this series of studies was to provide systematically

\footnotetext{
${ }^{1}$ Department of Community Medicine Lead Epidemiologist, Tropical Disease Research Unit, Faculty of Medicine and Allied Sciences, Rajarata University of Sri Lanka.
}

collected scientifically sound data on the epidemiology, etiological agent, clinical presentations, sequelae, risk factors and validity of diagnostic approaches of leptospirosis for control and prevention of leptospirosis in Sri Lanka. Lessons learnt from the outbreak investigation of leptospirosis in 2003, investigation of largestever outbreak reported in Sri Lanka in 2008, the flood associated outbreak of leptospirosis in 2011 in Anuradhapura and the first ever recreational associated leptospirosis reported from Sri Lanka in 2012 is presented here. Further, the utility of surveillance case definition, quantitative polymerase chain reaction (PCR) methods and multi locus sequence typing (MLST) typing methods which were field tested using the samples from these outbreaks are also described.

These studies conformed to the Helsinki Declaration and to local legislation. All participants gave informed consent to participate. Ethical clearance was obtained from the Ethics Review Committees of Faculty of Medicine, University of Peradeniya and Faculty of Medicine and Allied Sciences, Rajarata University of Sri Lanka. Throughout this series of studies, a set of definition as described by LERG was used to maintain the comparability of data ${ }^{18}$. These definitions are essential in leptospirosis due to the problems of clinical diagnosis of leptospirosis ${ }^{19}$.

\section{Investigation of leptospirosis outbreak in $2003^{20}$}

To investigate the leptospirosis outbreak in 2003 we used frozen samples from a fever surveillance study, which was conducted in teaching hospital Kandy. We performed immunoglobulin $M$ (IgM) enzymed linked immunosorbant assay (ELISA) on all suspected cases of leptospirosis and the positive samples were confirmed using microscopic agglutination test (MAT) with a broad panel of serovars. Twenty-two reference strains of Leptospira were included in the MAT panel. In this study, 473 suspected cases were investigated and 74 $(15.6 \%)$ were labelled as probable cases after IgM ELISA test. Around one third (35.1\%) of these patients were either housewives or unemployed males. Manual labourers accounted for $14.9 \%$ of the sample and agricultural workers or farmers $(10.8 \%)$ and soldiers $(5.4 \%)$ were the other occupation groups commonly affected. Anti leptospira antibodies were detected in 31 patients in MAT analysis. 
In this study, predominantly reacting serovars were serovar medenensis $(n=4)$ and hardjo $(n=3)$. In addition, one sample reacted only in serovar canicola showing a possible infection through dogs. Previous studies have identified serogroup icterohaemorrhagia as the commonly circulating serovar causing human infection in Sri Lanka ${ }^{21}$ which was not observed in 2003 outbreak in central Sri Lanka. The leptospira serovar hardjo, which was common in this study sample, is usually harboured by cattle and buffalo and the serovar canicola is typically found in dogs. Our study raised the probability that peri-domestic animals other than rodents may play an important role in transmission of human leptospirosis in Sri Lanka.

\section{Investigation of leptospirosis outbreak in 2008}

During the outbreak of leptospirosis in 2008, a hospital-based study was conducted in the districts of Kegalle, Kandy, and Matale in Sri Lanka. The study population consisted of all patients with acute febrile illness, confirming to the case definition of leptospirosis admitted to medical wards in the three main hospitals in selected districts. Socio-demographic profiles, exposure histories and environmental data were collected using a pre-tested, interviewer-administered, questionnaire. All cases were followed up until the end of the hospital stay to collect data on complications and sequelae and a follow up visit was arranged to collect convalescent phase serum sample. Acute and convalescent serum samples and acute whole blood samples were collected from all suspected patients. IgM ELISA testing ${ }^{26}$ was carried for all cases. MAT was performed by the WHO/FAO/OIE Collaborating Centre for Reference \& Research on Leptospirosis, Queensland, Australia. Molecular diagnosis was carried out at the Oxford-Mahidol Welcome Trust Research Unit, Bangkok, Thailand and at George Palade Laboratories of the School of Medicine, University of California, San Diego.

Of 746 fever patients admitted to the hospitals during the study period, 401 patients with possible leptospirosis were enrolled in the study. Convalescent serum samples were available from $167(41.6 \%)$ patients. MAT and PCR test confirmed 112 (27.9\%) cases of leptospirosis. Another $57(14.2 \%)$ cases were positive for ELISA and considered as probable cases. Of these confirmed cases, data were missing for one case and only 111 confirmed cases were subjected to further analysis. Of the PCR positive samples, DNA sequencing was carried out using a single tube nested PCR to amplify a region of the 16 s rDNA gene specific to pathogenic and intermediate Leptospira spp. on 32 samples. The deduced Leptospira species were $L$. interrogans ${ }^{29}$ and L.weilli ${ }^{3}$.
The classical clinical picture of leptospirosis was not observed in more than $50 \%$ of the cases in this study sample. Exposure of confirmed cases showed that $55(49.5 \%)$ had a single type of exposure, attributable to the present condition. Another 48 (43.2\%) cases had multiple types of exposures, making the assessment of the exact point of exposure difficult. $77.4 \%$ patients reported exposure in paddy field. Eight patients $(7.2 \%)$ could not recall any occasions, that they had any probable exposures, or outdoor work.

Acute renal failure was the most common complication observed in this series. Sixteen (22.8\%) patients had serum creatinine more than $1.5 \mathrm{~mol} / \mathrm{L}$ and seven of these cases showed acute bilateral renal paranchymal disease on ultrasound scan. Myocarditis was observed among 11 (7.1\%) patients and another $6(3.9 \%)$ patients had heart failure. Hyperbilirubinemia and pulmonary haemorrhage was not observed in this patient population. Case fatality rate of this study population was $1.9 \%$ and all three patients had fulminant myocarditis.

Of the clinical feature assessed at the hospital admission, oliguria [odds ratio $(\mathrm{OR})=4.1495 \%$ confidence interval $(\mathrm{Cl}) 1.003$ - 17.261], jaundice (OR $=5.13$ and $95 \% \mathrm{Cl} 1.149-28.003)$, and arrhythmias $(\mathrm{OR}=5.774$ and $95 \% \mathrm{Cl} 1.001-34.692)$, were the significant predictors of myocarditis and acute renal failure and none of the laboratory investigations could predict the two complications.

This study was the largest prospective study done on leptospirosis in Sri Lanka in recent years. We provided sufficient evidence to show that the reservoirs for leptospirosis in Sri Lanka are not limited to rodents. This knowledge is important in prevention programmes, specially where the knowledge on leptospirosis still is poor $^{27}$. The frequency of leptospirosis presenting with undifferentiated fever and the absence of the classical clinical picture of leptospirosis in this study shows the necessity for rapid and on site diagnosis for better patient care.

\section{Investigations post flood outbreak of leptospirosis in $2011^{28}$}

In 2011 a large outbreak of fever was observed in Anuradhapura just after heavy flooding in the area. Clinical diagnosis of leptospirosis outbreak was made and using the same protocols as for 2008 outbreak, we investigated this outbreak.

Total of 96 clinically suspected cases were studied and 32 (33.3\%) were confirmed using qPCR. These 32 cases were widely distributed within the 
district. Based on phylogenetic analysis of the $16 \mathrm{~S}$ rRNA gene, L. kirschneri (20), L. borgpetersenii (2) and $L$. Interrogans (1) were detected as species responsible for the outbreak in 2011. We also detected three dengue and one Hanta virus infection mimicking typical clinical features of leptospirosis.

A high level of complication rate was detected with $78.1 \%$ confirmed patients having thrombocytopenia $(<150,000 / \mu \mathrm{L})$ and in five $(15.6 \%)$, platelet count dropped to less than $20,000 / \mu \mathrm{L}$. Acute renal failure $(21.9 \%)$ with elevated blood urea over $40 \mathrm{mg} / \mathrm{dL}$ (53.1\%) and elevated liver enzymes (SGPT/SGOT) $(46.9 \%)$ were also common. Five patients had myocarditis and three of them required inotropic drugs and two required positive pressure ventilation. Pulmonary heamorrage was suspected among three patients and there were no fatalities during this outbreak.

During this outbreak we used a battery of investigations to characterise the leptospirosis like fever and confirmed that dengue and hanta virus infections may mimic leptospirosis and this could lead to clinical misjudgement.

\section{Investigations of leptospirosis associated with white water rafting in $2012^{29}$}

Recreational activity related leptospirosis is common among travellers from high-income countries. In countries like Sri Lanka, it has never ben reported in scientific literature. We received a request from a factory in Mihinthale, Sri Lanka to investigate an outbreak of fever among 20 office staff members. On $07^{\text {th }}$ September 2012, all of them participated in white water rafting and camping along the Kelani River in Kithulgala. Several of the office staff experienced fever approximately two weeks after the rafting trip and were treated in outpatient departments. One patient was having positive Leptospira IgM ELISA. We carried out outbreak investigation and the disease confirmation using MAT was done at MRI.

Of the 17 participants we investigated, four had moderate to severe febrile illness lasting 3 to 7 days. Another two had mild self-limiting fever. All six fever patients had myalgia and headache, five had prostration, two had mild jaundice and one had conjunctival suffusion. None of them had oliguria, anuria, dyspnoea, or other features of end organ failures. Two febrile patients and one non-febrile patient (who had experienced a mild headache, myalgia and anorexia) had a MAT titter of $1 / 400$. All other participants $(n=13)$ also showed anti-leptospiral antibodies; 9 with a titre of $1 / 200$ and 3 with a titre of $1 / 100$. The two participants that were not investigated, had positive leptospirosis IgM ELISA test.

\section{Validity and utility of different diagnostic methods in leptospirosis}

\section{Validity of WHO surveillance case definition ${ }^{30}$}

Even though the surveillance case definition proposed by the WHO is widely in use, a systematic assessment of the validity of this case definition was not available in global literature. We validated the surveillance case definition of leptospirosis among a sample of patients with undiagnosed acute febrile illness who were admitted to medical wards of Teaching Hospital Kandy, in 2007-8. The subjects were screened to diagnose leptospirosis according to leptospirosis surveillance case definition. MAT was performed using patoc-1 live antigen at MRI.

A total of 123 patients were recruited and 73 had clinical features compatible with surveillance case definition out of which 57 patients had positive MAT results. Total number of MAT positive cases was 62 , including five false negatives. Sensitivity, specificity, positive and negative predictive values of the surveillance case definition was $91.9 \%, 73.8 \%, 78.1 \%$ and $90.0 \%$ respectively.

Though this study has a major limitation due to not using the gold standard for comparison, preliminary data on validity of WHO case definition was provided to the global community through this study.

\section{Utility of quantitative PCR as a point of care diagnostic test ${ }^{31}$}

Quantitative polymerase chain reaction ( $q P C R$ ) has the potential to provide accurate and timely diagnosis for leptospirosis at the point-of-care in endemic areas. We studied optimal sample types for qPCR, timing of sampling, and clinical manifestations in relation to quantitative leptospireamia. A new qPCR assay using pathogenic Leptospira-specific 16S rRNA gene Taqman primers and an optimized temperature stepdown protocol was developed and used to analyze patient blood samples from 2008 outbreak. Serum was compared to whole blood as sample source. Quantitative leptospiremia was compared to clinical manifestations of leptospirosis and outcome.

Among the 381 cases studied, qPCR was positive up to the day 15 of onset of fever showing a high window of positivity. We compared the qPCR and acute sample MAT results (acute blood sample-obtained within 15 days of fever) to determine the utility of these tests as point of care diagnostics. Of the 73 confirmed samples tested, $36(47 \%)$ were qPCR positive while only 13 $(17 \%)$ had a diagnostic MAT titre $(>=1 / 400)$.

Bacterial load in serum/blood ranged from $10^{2}$ to $10^{6}$ Leptospira/mL among 58 positive cases. Median 
bacterial load was 9,577 Leptospira/mL (interquartile range(IQR)) 4,623 to $49,580 / \mathrm{mL}$ ). Patients with positive qPCR results were compared to identify any association between bacterial load and clinical manifestations. Median leptospiral load for un-complicated, renal failure, myocarditis, and multi-organ failure patients were 8,616 (IQR 4,611-41,727), 11,007 (IQR 1,102-17,417), 36,100 (IQR 11,162-104,067), and 15,882 (IQR 7,216-36,871) Leptospira/mL, respectively. Though the median bacterial load was lowest in uncomplicated group, bacterial load was not statistically different from other outcome categories (MannWhitney $U$ test, $p=0.59)$. More than $40 \%(n=17)$ of uncompli-cated cases had a bacterial load $>104 / \mathrm{mL}$. Among 40 male patients positive for qPCR, median leptospiral load was 15,640 Leptospira/mL (IQR 6,05353,980 Leptospira/mL) and this was significantly (Mann-Whitney $U$ test, $p=0.022$ ) higher than that of female patients (median 5,611, IQR 3,891 - 14,383 Leptospira/mL).

In this study of direct clinical application of molecular based techniques, the effectiveness of qPCR in terms of sensitivity, the longer window of positivity for qPCR in clinical samples, the ability to speciate the infection using molecular methods without culture and isolation and the optimal sample type for qPCR diagnosis were demonstrated. Further, the low sensitivity of MAT in comparison to new qPCR method in diagnosing acute leptospirosis was also confirmed. qPCR showed high potential as a point of care diagnostic test.

\section{Utility of multi locus sequence typing (MLST) in molecular characterization of infecting Leptospira species $^{32}$}

Culture isolation of Leptospira is insensitive and may take up to several months for a definitive result, and characterization of the infecting strain of leptospirosis, especially in outbreak investigations is difficult in tropical countries, where there are no resources to carry out extensive investigations. Recently, sequencebased typing techniques [including MLVA, MLST, etc.] have emerged as alternatives to serotyping ${ }^{33}$. MLST has typically required isolation of Leptospira to provide sufficient DNA for analysis. Nonetheless, because of difficulties in obtaining isolates of Leptospira from clinical samples (expense, expertise, problems with contamination and intrinsically poor isolation rates), being able to identify infecting Leptospira in clinical samples has a high priority for both clinical, epidemiological and basic science purposes. We used a previously published MLST approach to identify the infecting strain in serum and whole blood specimens collected during a 2008 outbreak of leptospirosis in Sri Lanka.

Of the 58 qPCR positive samples tested, 12 provided interpretable data. Three samples had complete MLST profiles i.e. sequences from all seven loci (Table 1) allowing tentative serovar assignment. Of the specimens from which we obtained incomplete MLST profiles, one shared two (of two) alleles with L. interrogans serovar Geyaweera strain Geyaweera, which is unique to Sri Lanka. All other strain types (STs) were similar to ST1, L. interrogans serovar Lai.

Table 1. Definitions of leptospirosis used in the series of studies

\section{Laboratory-confirmed cases of leptospirosis (definitive cases)}

A patient with clinical signs and symptoms consistent with leptospirosis and any one of the following:

1. fourfold increase in Microscopic Agglutination Test) (MAT) titre in acute and convalescent serum samples; negative acute and positive (MAT titre $>1: 100$ ) convalescent serum samples

2. MAT titre $\geq 1: 400$ in single or paired serum samples; pathogenic Leptospira species DNA detected by PCR

\section{Probable cases of leptospirosis}

A patient with clinical signs and symptoms consistent with leptospirosis and one of the following: presence of IgM antibodies by enzyme-linked immunosorbent assay (ELISA); MAT titre $\geq 1: 400$ in single or paired serum samples using genus specific Leptospira strain patoc

\section{Surveillance case definition}

Acute febrile illness, with headache, myalgia, and prostration associated with any of the following: conjunctival suffusion/haemorrhage; meningial irritation; anuria/ oliguria/ haematuria/ proteinuria; hemorrhage-intestinal bleeding, lung bleeding or purpuric rash; cardiac arrhythmias/failure; Plus:

History of exposure to infected animals/environment contaminated with animal urine 


\section{Possible cases of leptospirosis (used only for 2008 outbreak investigation)}

Any acute febrile patient with any one of the following major symptoms:

headache; myalgia; prostration

associated with any of the following signs (at least one):

conjunctival suffusion / conjunctival hemorrhage; meningial irritation; anuria or oliguria / proteinuria /

haematuria; jaundice; hemorrhages; purpuric skin rash; cardiac arrhythmia or failure

or, any other patient suspected and treated as a leptospirosis patient by a treating physician

This study was the first report of using a full MLST-typing scheme to directly identify infecting Leptospira in human clinical specimens. This study clearly showed that the MLST can be used to directly identify infecting Leptospira strains in blood samples obtained during acute illness without the need for culture isolation but shows important limitations related to bacterial load. The data presented here suggest that ST1 was the predominant infecting ST among the study population during the 2008 outbreak of leptospirosis in Sri Lanka. Based on serology, ST1 appears to be $L$. interrogans serovar Lai, previously reported to be a highly virulent leptospire and for the first time reported to be present in Sri Lanka. However, in the Sri Lanka setting, serovar Lai (disease caused by the strain that appears to be similar to Lai) seemed to be associated with both severe and non-severe disease which has not been reported previously.

Table 2. Frequency of MAT antibody titres to the test panel of Leptospira spp. Among 87 MAT positive cases of leptospirosis during 2008 outbreak in Kegalle, Kandy and Matale, Sri Lanka

\begin{tabular}{|c|c|c|c|c|c|}
\hline Species & Serogroup & Serovar & $n$ & $\%$ & $\begin{array}{l}\text { Range of antibody } \\
\text { titers in } M A T^{*}\end{array}$ \\
\hline L. interrogans & Pyrogenes & Pyrogenes & 25 & 28.7 & $200-6400$ \\
\hline L. borgpetersenii & Javanica & Ceylonica & 10 & 11.5 & $100-3200$ \\
\hline L. interrogans & Sejroe & Hardjo & 10 & 11.5 & $100-6400$ \\
\hline L. interrogans & Hebdomadis & Hebdomadis & 10 & 11.5 & $100-6400$ \\
\hline L. borgpetersenii & Sejroe & Geyaweera & 6 & 6.9 & $400-3200$ \\
\hline L. interrogans & Loisiana & Lanka & 5 & 5.8 & $100-3200$ \\
\hline L. interrogans & Autumnalis & Weerasinghe & 4 & 4.6 & $400-6400$ \\
\hline L. santarosai & Autumnalis & Alice & 2 & 2.3 & $400-3200$ \\
\hline L. interrogans & Australis & Australis & 2 & 2.3 & $200-400$ \\
\hline L. interrogans & Autumnalis & Autumnalis & 2 & 2.3 & $100-800$ \\
\hline L. interrogans & Icterohaemorrhagiae & Copenhageni & 2 & 2.3 & $1600-3200$ \\
\hline L. interrogans & Pomona & Pomona & 2 & 2.3 & $100-400$ \\
\hline L. kirschneri & Grippotyphosa & Ratnapura & 2 & 2.3 & $200-1600$ \\
\hline L. interrogans & Canicola & Canicola & 2 & 2.3 & $100-200$ \\
\hline L. interrogans & Bataviae & Bataviae & 1 & 1.2 & 800 \\
\hline L.weilii & Celledoni & Celledoni & 1 & 1.2 & 200 \\
\hline L.weilii & Sarmin & Sarmin & 1 & 1.2 & 400 \\
\hline
\end{tabular}

* the results shown represent the highest titer for each patient 
Table 3. Clinical profile of confirmed cases during 2008 outbreak of leptospirosis

\begin{tabular}{lcclcc}
\hline Clinical symptoms & $n$ & $\%$ & Clinical signs & $n$ & $\%$ \\
\hline Headache & 93 & 83.8 & Conjunctival suffusion & 47 & 42.3 \\
Myalgia & 84 & 75.7 & Jaundice & 35 & 31.5 \\
Arthralgia & 80 & 72.1 & Muscle tenderness & 34 & 30.6 \\
Prostration & 69 & 62.2 & Hepatomegaly & 17 & 15.3 \\
Nausea / vomiting & 72 & 64.9 & Neck stiffness & 11 & 9.9 \\
Anorexia & 59 & 53.6 & & & \\
Chills & 40 & 36.0 & & & \\
Oliguria & 42 & 37.8 & & & \\
Abdominal pain & 32 & 28.8 & & & \\
Diarrhea & 26 & 23.4 & & & \\
Shortness of breathing & 13 & 11.7 & & & \\
Mental confusion & 6 & 5.4 & & & \\
\end{tabular}

\section{Conclusions}

This series of studies widely expanded the knowledge on human leptospirosis in Sri Lanka to identify the epidemiology, etiological agents, varying clinical presentations and sequelae. The lessons learnt here are applied in the national control programme and also this research is used in global disease estimates as a model for tropical, resource poor countries.

Further, these clinical and epidemiological studies linked with molecular typing provided early evidence for probable microgeographical changes in lepto spirosis. The wide variability of clinical outcomes, biochemical findings and infecting serotype in different outbreaks in different geographical locations needs further investigations in order to understand the microgeographic variation of this important disease. Human leptospirosis is caused by spirochetes which belongs to 10 species and more than 240 serovars. Number of animal reservoir that could harbour Leptospira is countless. In tropical countries, environmental sources of leptospirosis are abundant. All these factors can lead to extensive microgeographical variations even within countries. These variations provide multitude of enabling environment to different strains of Leptospira, which causes varying types of clinical disease. The understanding on these variations are essential in designing control and preventive activities and management strategies for probable different clinical outcomes.

\section{Acknowledgment}

I acknowledge the guidance of all my supervisors Dr. Ananda Amarasinghe, Prof. Vasanthi Thevanesam,
Prof. D. B. Nugegoda, Prof. Sharon Peacock and Prof. Joshep M Vinetz. Dr. Paba Palihawadana provided financial support through WHO funding for initial work and Oxford Mahidol Research Unit also supported the field data collection during 2008 outbreak. The major funding was through US Public Health Service Grants 1R01TW05860, 1U01AI075420.

1K24AI068903, 5R25GM083275, and 1D43TW007120. Ms. Thamarasi Senarathna, Mr. Athula Kumara and Dr. Sahan Perera were involved in the 2008 outbreak investigation procedure. My collaborators, Janjira Thaipandungpunit and her colleagues from Mahidol University provided the initial support for molecular diagnosis and Lee Smyth and his colleagues at WHO leptospirosis reference laboratory in Queensland carried out MAT testing. I appreciate the contribution of all other co-authors in all published work. Michael M Matthias, Anjelo Moreno and Jason Lehman helped extensively in molecular work. Thilini Agampodi helped in every aspect of my work throughout this period providing constant support, encouragement and scientific inputs to my work. I finally appreciate all the hospital staff, lab staff and other supportive staff who were involved in these activities.

\section{References}

1. WER, Leptospirosis worldwide.1999. Wkly Epidemiol Rec 1999; 74(29): 237-42.

2. Cachay ER, Vinetz JM. A global research agenda for leptospirosis. J Postgrad Med, 2005; 51(3): 174-8.

3. World Health Organization. Report of the first meeting of leptospirosis burden epidemiology reference group. 2010, World Health Organization Geneva. 1-34. 
4. Rajasuriya K, Somasunderam M, Nagaratnam N. Leptospirosis in Ceylon. J Trop Med Hyg 1959. 62: 205-10.

5. Nagaratnam N, Weerasinghe WM, Fernandopulle M, Leptosiprosis (anicteric) as a cause of long and short term pyrexia in the Kegalla district (Ceylon). Ceylon Med J 1967; 12(4): 225-8.

6. Thirunavukkarasu K, De Silva VN, Amarasinghe G. Leptospirosis in Ceylonese children. Ceylon Med J 1967; 12(4): 202-5.

7. Nityananda K, Sulzer CR. A new serotype of Leptospira belonging to the autumnalis serogroup. J Trop Med Hyg 1971; 74(8): 184-6.

8. Ramachandran S, Rajapakse CN, Perera MV. Changing patterns in leptospirosis in Sri Lanka. Ceylon Med J 1974; 19(3): 142-9.

9. Rajasuriya $\mathrm{K}$, et al. Leptospirosis in Ceylon: a Clinical Study. Ceylon Med J 1964; 93: 136-53.

10. Ramachandran $\mathrm{S}$, et al. Patterns of acute renal failure in leptospirosis. J Trop Med Hyg 1976; 79(7): 158-60.

11. Epidemiology Unit, Leptospirosis Surveillance Report - 2003. Epidemiological Bulletin Sri Lanka 2004; 45(2): 16.

12. Agampodi, S, Peacock SJ, Thevanesam V. The potential emergence of leptospirosis in Sri Lanka. Lancet Infect Dis 2009; 9(9): 524-6.

13. Epidemiological Unit, Selected notifiable diseases reported by Medical Officers of Health. Weekly Epidemiological Report 2009; 36(1): 4.

14. Pappas $\mathrm{G}$, et al. The globalization of leptospirosis: worldwide incidence trends. Int J Infect Dis 2008; 12(4): 351-7.

15. Agampodi SB, et al. Timelines and completeness of notification data in the $\mathrm{MOH}$ area in Beruwala. CMJ 2008; 53: 26.

16. Chang $A A$, et al. A case of primary neuroleptospirosis. Ceylon Med J 2003; 48(4): 143-4.

17. Agampodi SB. Doxycycline chemoprophylaxis for leptospirosis - a brief review. Sri Lanka Journal of Medicine 2008; 17(1): 1-3.

18. Agampodi SB. Case definitions in Leptospirosis: a note to Sri Lankan researchers. Sri Lankan Journal of Infectious Diseases 2012; 2(2): 55-7.

19. Agampodi SB, Re: Severe Leptospirosis - a case series and review. Sri Lankan Journal of Anaesthesiology 2012; 20 (1): 62-3.

20. Agampodi SB, et al. A preliminary study on prevalent serovars of leptospirosis among patients admitted to teaching hospital, Kandy, Sri Lanka. Indian J Med Microbiol 2008; 26(4): 405-6.
21. Babudeiri B, Jagels G. Serological research on the presence of leptospirosis in Ceylon. Ceylon Med J 1962; 1964(93): 136-53.

22. Agampodi S. Epidemiology of leptospirosis among patients admitted to selected tertiary care hospitals in three endemic districts in Sri Lanka, during the 2008 epidemic, in Community Medicine 2010; University of Colombo: Colombo.

23. Agampodi SB, Nugegoda DB, Thevanesam V. Determinants of leptospirosis in Sri Lanka: Study Protocol. BMC Infect Dis 10(1): 332.

24. Agampodi SB, et al. Leptospirosis Outbreak in Sri Lanka in 2008: Lessons for Assessing the Global Burden of Disease. Am J Trop Med Hyg 85(3): 471-8.

25. Dassanayake DL, et al. Predictors of the development of myocarditis or acute renal failure in patients with leptospirosis: an observational study. BMC Infect Dis 2012; $12: 4$.

26. Levett PN, Branch SL. Evaluation of two enzyme-linked immunosorbent assay methods for detection of immunoglobulin $\mathrm{M}$ antibodies in acute leptospirosis. Am J Trop Med Hyg 2002; 66(6): 745-8.

27. Agampodi SB, et al. Do People Know Adequately about Leptospirosis? A Knowledge Assessment Survey in Post-outbreak Situation in Sri Lanka. Int J Prev Med 2010; 1(3): 158-63.

28. Agampodi SB, et al. Regional differences of leptospirosis in Sri Lanka: observations from a flood-associated outbreak in 2011. PLoS Negl Trop Dis 2014; 8(1): e2626.

29. Agampodi SB, et al.Outbreak of leptospirosis after whitewater rafting: sign of a shift from rural to recreational leptospirosis in Sri Lanka? Epidemiol Infect 2014; 142(4): 843-6.

30. Dassanayake DL, et al. Evaluation of surveillance case definition in the diagnosis of leptospirosis, using the Microscopic Agglutination Test: a validation study. BMC Infect Dis 2009; 9: 48.

31. Agampodi SB, et al. Utility of quantitative polymerase chain reaction in leptospirosis diagnosis: association of level of leptospiremia and clinical manifestations in Sri Lanka. Clin Infect Dis 2012; 54(9): 1249-55.

32. Agampodi SB, et al. Utility and limitations of direct multilocus sequence typing on qPCR-positive blood to determine infecting Leptospira strain. Am J Trop Med Hyg 2013; 88(1): 184-5.

33. Levett PN. Sequence-based typing of leptospira: epidemiology in the genomic era. PLoS Negl Trop Dis 2007; 1(2): e120. 